University of Wollongong

Research Online

Faculty of Social Sciences - Papers (Archive) Faculty of Arts, Social Sciences \& Humanities

2010

Adaptation of an interview-based protocol to examine close relationships between children with developmental disabilities and peers

Amanda A. Webster

University of Wollongong, awebster@uow.edu.au

Mark Carter

Macquarie University

Follow this and additional works at: https://ro.uow.edu.au/sspapers

Part of the Education Commons, and the Social and Behavioral Sciences Commons

Research Online is the open access institutional repository for the University of Wollongong. For further information contact the UOW Library: research-pubs@uow.edu.au 


\title{
Adaptation of an interview-based protocol to examine close relationships between children with developmental disabilities and peers
}

\author{
Abstract \\ The purpose of this study was to determine whether an interview protocol, based on the Friendship \\ Quality Questionnaire, could be adapted to examine the close relationships of children with \\ developmental disabilities in an inclusive school setting. Twenty-five children with developmental \\ disabilities aged between approximately 5 and 12 years participated and their relationships with 74 peers \\ were examined. Several adaptations to the procedures and interview instrument were evaluated, including \\ gathering interview data from multiple sources and the development of a short form of the interview \\ questionnaire. Overall, the adaptations to procedures used in the current study appeared successful in \\ catering for the wide range of abilities and ages among respondents. This was reflected in the reliability \\ of children's responses, high response rates on the short interview form, and good correspondence \\ between the short and full interview forms. The described adaptations were successful in eliciting \\ information on aspects of children's relationships that might not have been obtained using a traditional \\ interview instrument. This opens the way for further detailed quantitative evaluation of relationships \\ between children with developmental disabilities and peers in inclusive settings.
}

\section{Keywords}

children, developmental, disabilities, peers, between, relationships, close, examine, protocol, interview, adaptation

\section{Disciplines}

Education | Social and Behavioral Sciences

\section{Publication Details}

Webster, A. A. \& Carter, M. (2010). Adaptation of an interview-based protocol to examine close relationships between children with developmental disabilities and peers. Australasian Journal of Special Education, 34 (1), 79-94. 


\title{
Adaptation of an Interview-Based Protocol to Examine Close Relationships Between Children With Developmental Disabilities and Peers
}

\author{
Amanda A. Webster and Mark Carter \\ Macquarie University Special Education Centre, Sydney, Australia
}

\begin{abstract}
$T^{1}$ he purpose of this study was to determine whether an interview protocol, based on the Friendship Quality Questionnaire, could be adapted to examine the close relationships of children with developmental disabilities in an inclusive school setting. Twenty-five children with developmental disabilities aged between approximately 5 and 12 years participated and their relationships with 74 peers were examined. Several adaptations to the procedures and interview instrument were evaluated, including gathering interview data from multiple sources and the development of a short form of the interview questionnaire. Overall, the adaptations to procedures used in the current study appeared successful in catering for the wide range of abilities and ages among respondents. This was reflected in the reliability of children's responses, high response rates on the short interview form, and good correspondence between the short and full interview forms. The described adaptations were successful in eliciting information on aspects of children's relationships that might not have been obtained using a traditional interview instrument. This opens the way for further detailed quantitative evaluation of relationships between children with developmental disabilities and peers in inclusive settings.
\end{abstract}

Keywords: friendship, relationships, children, developmental disabilities, peers, interview

In 1953, Henry Stack Sullivan described the importance of social relationships, and friendship in particular, in the lives of preadolescent children. Since then, researchers have invested considerable effort trying to define friendship, measure its components and determine the quality of friendships between two individuals (Berndt \& Perry, 1986; Gottman, 1983; Mannarino, 1980; Newcomb \& Bagwell, 1996). It has also been established that parents and teachers feel that fostering friendships is especially important for children with disabilities (Hamre-Nietupski, 1993; Hamre-Nietupski, Hendrickson, Nietupski, Sasson, \& Shokoohi-Yekta, 1993; Overton \& Rausch, 2002). The development of social relationships has been offered as an important justification for the inclusion of children with more significant disabilities in

Address for correspondence: Amanda Webster, Macquarie University Special Education Centre, Macquarie University, NSW 2109, Australia. E-mail: Seapig@octa4.net.au 
education settings alongside their typically developing peers (Falvey \& Rosenberg, 1995; Grenot-Scheyer, Fisher, \& Staub, 2001; Moore, 1998; Villa \& Thousand, 1995). Others have argued that the investigation of these relationships is imperative as children with disabilities may not form the same types of relationships as would generally be found between typically developing children (Howes, 1983; HurleyGeffner, 1995).

Although considerable research has been dedicated to describing the friendships of typically developing children (e.g., Bukowski, Hoza, \& Boivin, 1994; Furman, 1996; Newcomb \& Bagwell, 1996; Parker \& Asher, 1993), research on the relationships and friendships of children with disabilities has been much more limited. Recently, Webster and Carter (2007) reviewed the extant literature on relationships between children with developmental disabilities and peers. Thirty-six studies were examined and included target populations with developmental disabilities such as cerebral palsy, autism, intellectual impairment, and multiple disabilities. An analysis of the research questions indicated that the focus of the studies was extremely varied and somewhat patchy in its scope. Much of the research explored the differing impact of environments (inclusive versus segregated) (Buysse, Goldman, \& Skinner, 2002) or of the individual characteristics of the children with disabilities (Guralnick, 1997; Guralnick \& Groom, 1988; Strain, 1984) on the relationships that were formed. A number of studies investigated the correlation between the behaviour of the child with disabilities and the development of social relationships with peers (Bauminger \& Kasari, 2000; Buysse, 1993; Field, 1984; Guralnick, 1999; Hall, 1994; Hall \& Strickett, 2002). Another body of research has investigated the behaviours of children with disabilities while involved in specific and time-limited interactions with peers (Evans, Salisbury, Palombaro, Berryman, \& Hollowood, 1992; Lee, Yoo, \& Bak, 2003; Siperstein, Leffert, \& Wenz-Gross, 1997). In all of the aforementioned studies, however, the focus was not necessarily on describing the specific components of relationships, as has been the case with research involving typically developing children. Many of the studies purporting to examine the friendships of children with disabilities have employed sociometric measures that actually determined the reported play preferences of only one child in a dyad (e.g., Evans et al., 1992) or measured peer acceptance, rather than friendship (e.g., Buysse, Nabors, Skinner, \& Keyes, 1997; Fujiki, Brinton, Hart, \& Fitzgerald, 1999; Nabors, 1997).

Although a small number of researchers (Bauminger \& Kasari, 2000; Chamberlain, Kasari, \& Rotherham-Fuller, 2007; Cuckle \& Wilson, 2002; Heiman, 2000; Kennedy \& Itknonen, 1994; Zetlin \& Murtaugh, 1988) have used direct methods to analyse the features of relationships between children with disabilities and peers, the most descriptive information has been provided by a group of qualitative investigations of children with developmental disabilities in inclusive settings (Fisher, 2001; Meyer et al., 1998; Richardson \& Schwartz, 1998; Salisbury \& Palombaro, 1998; Staub, 1998). These researchers have employed a combination of observations and open-ended interviews to present a preliminary picture of several relationships and friendships that they identified in a number of schools.

In typically developing children, descriptions of the features of close relationships have often relied on questionnaire measures. In particular, variations on the Friendship Quality Scale, which was originally developed by Bukowski et al.(1994) have provided a rich and detailed insight into the nature of close relationships (Asher, Parker, \& Walker, 1996; Bukowski et al., 1994; Ladd, Kochenderfer, \& Coleman, 1996; Newcomb \& Bagwell, 1996; Parker \& Asher, 1993). This methodology has been applied 
to children with learning disabilities (Wiener \& Schneider, 2002) and to a limited extent with children with high functioning autism (Bauminger \& Kasari, 2000; Chamberlain et al., 2007). It has not, however, been used to evaluate the relationships of children with more significant levels of disability, specifically those with developmental disabilities, who are increasingly the focus of inclusion in schools. Freeman and Kasari (1998) have noted that instruments previously used to measure the friendships of typically developing children may have limitations for children with developmental disabilities, depending on the child's developmental level and communication skills. Thus, adaptations of such instruments will almost certainly be needed to accommodate the needs of students with developmental disabilities.

One particular difficulty is that some students with developmental disabilities may have impairments in cognitive or communication skills that seemingly preclude their participation in questionnaire-based research. Some previous researchers have relied on teacher or parent reports to evaluate the friendships of young children with disabilities (Buysse, 1993; Buysse \& Bailey, 1993; Buysse et al., 2002; Buysse et al., 1997; Guralnick, 1997; Guralnick, Connor, \& Hammond, 1995). This approach may have potential application to a wider range of children with developmental disabilities and may provide a more complete view of the character of close relationships. It has been noted, however, that teachers and parents may have different perceptions of the friendships examined than the children themselves (Buysse, 1993; Buysse et al., 1997). Freeman (Freeman 2000; Freeman \& Kasari, 1998) has argued that, rather than limiting the measurement to one person's perceptions or reported behaviours, the use of multiple respondents should present the most accurate picture of close relationships. As established by Weiner and Schneider (2002), the perspectives of both members of a relationship must be considered in order to determine whether these perspectives are reciprocal. Thus peers would also be important respondents.

Where students with developmental disabilities are able to attempt a questionnaire, the relatively complex nature of response options (most applications involve 5-point response scales) may present problems. The current research also included children with multiple and severe disabilities as well as a range of ages, including children in preschool. Thus some children may also have difficulties following instructions, and it is possible that younger or more distractible children may have problems completing a full questionnaire. As a result, modifications to both response scales and procedures may be needed to accommodate a wide variety of children with developmental disabilities.

Questionnaires previously developed to examine the relationships between typically developing children have been used for students with specific learning disabilities (Wiener \& Schneider, 2002) and high functioning autism (Bauminger \& Kasari, 2000; Chamberlain et al., 2007). To date, these questionnaires have not been systematically adapted for children with more significant cognitive or communication impairments across a wide range of ages and abilities. Thus, the primary objective of the current study was to determine whether a questionnaire protocol, based on the Friendship Quality Questionnaire developed by Parker and Asher (1993), could be adapted to describe the school-based close relationships of children with developmental disabilities aged between approximately 5 and 12 years. The adaptations included a range of procedural modifications, the use of multiple respondents to obtain a more complete dataset, and the development of a short-form questionnaire that was delivered by interview. 


\section{Methodology}

\section{Setting}

The research was conducted in public and private primary schools and preschools in Alice Springs, Australia.

\section{Selection of Target Students}

A letter was sent to all area primary schools detailing the basic parameters of the study and outlining the criteria for selection of the target students. Schools were asked to identify any student who: (1) had been identified as having a developmental disability, which was defined as a significant delay in adaptive behaviour and at least one other area of functional impairment such as cognitive or communication skills (Centre for Developmental Disability Studies, 2001; Developmental Disabilities Assistance and Bill of Rights Act 2000; National Association of Developmental Disabilities Councils, 2003; Northern Territory Government, 2005); (2) had a high level of educational need in that he/she had been identified by the Northern Territory Department of Education, Employment and Training as in need of individual assistance in order to access the curriculum; (3) had a record of regular attendance and/or would be present in school for the entire school year; (4) had not been identified as a child whose primary disability was a sensory impairment (i.e., impairments in hearing, vision, physical skills), or behaviour problem. These needs may have existed as a secondary issue to the developmental disability. In addition, children were excluded from the study if the primary diagnosis was a learning disability with average intellectual abilities, low achievement, and no corresponding significant delays in other areas or adaptive behaviour.

All ten area public and private primary schools agreed to participate but three private schools (two of which were very small) reported that they did not have any students who met the criteria. Participating schools nominated all students who met the criteria for the study. Parental consent was obtained and the names of nominated students were submitted to the researchers. Twenty-nine students were initially nominated. Another student was added by a school at a slightly later date.

\section{Target Students}

Parental consent was obtained for 25 students who were included in the final target population for data collection representing $83 \%$ of the children with developmental disabilities in inclusive schools in Alice Springs. Six of the selected students were in preschool (mean age $=5.4$, range 4.9 to 6.1 ), 12 were in the junior primary grades of transition through to grade 3 (mean age $=7.2$, range 5.1 to 9.4 ), and seven were in senior primary grades 4 through 6 (mean age $=10.9$, range 10.0 to 12.1 ). Target students were predominately male with four girls and 21 boys. It should be noted that males tend to predominate in prevalence figures for developmental disabilities (Bhasin, Brocksen, Avchen, \& Braun, 2006; Rice, 2007) and more importantly, that this ratio was the true representation of boys to girls in the target population in the Alice Springs area. Based on diagnostic reports of the 25 target students with a developmental disability, 13 students had a primary diagnosis of an intellectual disability (eight mild, four moderate, and one severe disability) with compounding disabilities in communication and motor skills. Six students had a primary diagnosis of an autistic spectrum disorder, one student had cerebral palsy, two students had severe communication disorders with compounding social-emotional and learning delays, and three students had unspecified developmental delays with deficits in 
multiple areas. The mean Adaptive Behavior Composite for target students as assessed on the Vineland Adaptive Behavior Scales: Classroom Edition (Sparrow, Balla, \& Cicchetti, 1985) was 64.60 (range 42 to 72 ).

\section{Nomination of Peers}

The wide range of ages and communication levels of the target population in the present study made it necessary to find a method of peer nomination that allowed those target students who were capable to participate in the peer selections. Thus, it was decided that a combination of teacher and target student nomination would be used. At the discretion of the teacher, older and more verbal students were asked to identify their three closest friends. In these instances, the students' choices were verified by the teacher. For the remaining target students, teachers were asked to nominate three friends or, if three friends could not be identified, children with whom the target student most frequently interacted. Target students were then asked if the three peers chosen by their teacher were their friends. At the same time, target students were asked if an additional three students, selected randomly from their class as distractors, were their friends. If a target student said that any of the peers identified by the teacher was not his/her friend, the teacher was asked to identify another close peer. This alternative nomination was again confirmed by asking the target student about the peer as well as a randomly selected peer as a distractor. All target students confirmed teachers' selections of peers with the exception of five who were nonverbal who did not confirm or deny teacher nominations.

\section{Data Collection}

An original set of 38 questions were taken from the Friendship Quality Questionnaire developed by Parker and Asher (1993). Parker and Asher's subscales of friendship included: 1) Validation and Caring, 2) Conflict Resolution, 3) Conflict, 4) Help and Guidance, 5) Companionship, and 6) Intimate Exchange. In addition, the researchers also considered that some areas were not adequately covered without the inclusion of some of the questions in the research conducted by Bukowski et al. (1994), whose earlier instrument (Bukowski, Hoza, \& Newcomb, 1987) formed the basis for Parker and Asher's (1993) Friendship Quality Questionnaire. The Friendship Quality Questionnaire (Parker \& Asher, 1993) and Friendship Qualities Scale (Bukowski et al., 1994) have been extensively researched and used by previous researchers to measure the relationships and friendships between typically developing children (Schneider, 1999; Schneider, Fonzi, Tani, \& Tomada, 1997) and between children with disabilities who were within the average range of intelligence (Bauminger \& Kasari, 2000; Chamberlain et al., 2007; Wiener \& Schneider, 2002; Wiener \& Tardif, 2004). Ten questions were additionally included from the Friendship Qualities Scale developed by Bukowski et al. It should be noted that minimal modifications were made from the original interview questions validated by Parker and Asher (1993) and Bukowski et al. (1994) and that all interview questions used in this research and discussed in this article were taken from the questionnaires developed by Parker and Asher (1993) and Bukowski et al. (1994). All questions used in the interview are presented in Table 1 along with the dimension with which they were associated, corresponding definitions, and a note on the source of the questions. It should be noted that the research discussed in this article was part of a larger study. The final instrument contained 77 questions. Twenty-nine of these questions were not pertinent to research discussed in this article.

As the instrument was to be used with children with developmental disabilities over a considerable age range, it was anticipated that obtaining direct information from some 


\section{TABLE 1}

Definitions of Dimensions, and Interview Questions

Dimension Interview question

\section{Companionship.}

Questions 1, 4, 36, 56, 65, 2, and 48 were taken from Parker \& Asher's (1993)

Companionship \& Recreation dimension: the extent to which the friends spend enjoyable time together inside school.
1, 4. Do you and

36. Do you and

56. Do you and

65. Do you and

2. Do you and school or on weekends?

48. Do you and do fun things together?

Companionship.

Questions $32 \& 40$ were taken from

Bukowski, Hoza, \& Boivin's (1994)

Companionship dimension: focuses on amount of voluntary time spent together.
32. Do you and

40. Do you and spend your free time together? just sit around and talk about things like school, sports, and things we like?
Validation and Caring.

Questions 3, 17, 22, 24, and 54 were taken from Bukowski, Hoza, \& Boivin's (1994)

Closeness dimension - affordances for feelings of acceptances, validation, and attachment, focus on the sense of affection or 'specialness' that the child experiences with a friend and the strength of the child's attachment or bond to the friend.

Validation and Caring.

Questions 11, 23, 76

$77,5,38,42,70$, and 78 were taken from Parker \& Asher's (1993) Validation \& Caring dimension: the degree to which the relationship is characterised by caring, support and interest. secrets?

3. Do you feel happy when you are with ___ ?

17. Are you happy for __ when he/she does a good job at something?

22. When you do a good job at something, is for you?

24. Do you think that is happy when he/she is with you?

54. If___ moved away, would you miss him/her?

11. Does _ tell you that you are good at things?

23. Do you tell that he/she is good at things?

76. Does_tell you that you are pretty clever?

77. Does __ stick up for you if other's talk behind your back?

5. Does_care about your feelings?

38. Do you think ___ does not tell others your

42. Would still like you if other kids didn't like you?

70. Does__ say 'I'm sorry' if he/she hurts your feelings?

78. Do you care about 's feelings?

Help and Guidance.

Questions 7, 29, and 61 were taken from Bukowski, Hoza, \& Boivin's (1994) Help dimension that was made up of 2 subcomponents of Aid and Protection from Victimization: 1) Indicate that mutual help and assistance are features of friendship relation; 2) Refer to friend's willingness to come to the child's aid if another child were bothering him/her.

\section{Help and Guidance.}

Questions 9, 50, 51,

$52,58,69,73,74$, and 75 were taken from Parker \& Asher's (1993) Help \& Guidance dimension: the extent of the friends' efforts to assist one another with routine or challenging tasks.
7. Would help you if you needed it?

29. Would your child help ___ if he/she needed it?

61. If other kids were bothering your child, would help him/her?
9. My child and share things with each other?

50. Does quicker? 51. Do you and
ways to do things help you with things so you can get done

52. Do you and ___ count on each other good ideas about games to play?

58. Do you and loan each other things?

69. Do you and to do things? happy

help each other with schoolwork?

pick each other as partners?

fter 
TABLE 1 (CONTINUED)

Definitions of Dimensions, and Interview Questions

\begin{tabular}{ll}
\hline Dimension & Interview question \\
\hline Help and Guidance. & $\begin{array}{l}\text { 73. Do you and __ help each other with jobs or other } \\
\text { things? }\end{array}$ \\
$\begin{array}{l}\text { 74. Do you and __ do special favors for each other? } \\
\text { 75. Do you and and special? }\end{array}$
\end{tabular}

Intimate Exchange.

Questions 12, 53, 55, and 59 were taken from Parker \& Asher's (1993) Intimate

Exchange dimension: the extent to which the relationship is characterised by disclosure of personal information and feelings.
12. Do you and

53. Do you and

55. Do you and

59. Do you talk to something that has happened to you? talk about things that make you sad? tell each other your problems? tell each other secrets? when you are mad about

Conflict.

Questions 10, 13, 33, 45, 57, 60, and 62 were taken from Parker \& Asher's (1993) Conflict \& Betrayal dimension: the extent to which the relationship is typified by argument, disagreement, annoyance, and mistrust.
10, 13. Do you and fight?
33. Do you and
45. Do you and
57. Do you and
60. Do you and
62. Do you think
tease each other? get mad at each other? bother each other? argue? doesn't listen to you?

\section{Conflict Resolution. \\ Questions 34, 37, 46 and 79 were taken from Parker \& Asher's (1993) Conflict Resolution dimension: the degree to which disagreements in the relationship are resolved efficiently and fairly through talking.}
34. If you and get mad at each other, do you talk about how to get over it?
37. Do you and a fight?
46. If you and ___ are mad at each other, do you talk about what would help to make you feel better?
79. Do you and

children would be difficult or impossible. Thus, a number of strategies were used to attempt to obtain as complete a dataset as possible. Interviews were conducted with all target students, nominated peers, teachers, and parents of target students. This approach, utilising multiple respondents, was employed in order to present the most complete and accurate picture of the relationships between target students and peers. As many of the children were very young, and some target students were nonverbal, interviews conducted with only the children might not have provided information on some relationships. Previous researchers (Buysse et al., 2002; Guralnick et al., 1995; Overton \& Rausch, 2002) have sometimes relied solely on the responses of teachers and/or parents to describe friendships of children in preschool or day care settings. Although information from students was extended by asking parents and teachers to provide information, the researchers in this study considered that while not all of the children could complete the full interview form, a sizeable number could provide some information on their perspective of the relationships, and that this information would be too relevant to be dismissed. In addition, a short interview form was developed that included the 10 questions considered to best reflect the salient aspects of the research question. Unfortunately, due to the complexity of the interview questions associated with Conflict Resolution, the researchers considered that young children and children with limited language would have difficulty providing an answer to these questions. Thus, no questions associated with Conflict Resolution were included on the short 
interview form. (It should be noted that Question 6/8 was not relevant to this study, but to another aspect of the overall research). The short interview form was used with all children in preschool and transition (preprimary) and with any target student whose teacher or parents considered would be unable to complete the full interview form. The 10 questions on the short interview form were also included as the first questions on the long interview form. Three of the first 10 questions were repeated for child respondents to assess reliability, making a total of 13 questions.

Questionnaires were delivered via interview by the first author. Interviews were conducted at schools for target students, nominated peers, teachers, and some parents. The remaining parent interviews were conducted at community locations such as homes and workplaces. Two parent interviews were conducted on the phone. Interview sessions lasted approximately 10 to 60 minutes depending on whether the short interview form or the full interview form was used. Except in one instance, interviews were administered in a single session. One nominated peer stated he was tired after 30 minutes and requested the interview be completed on the following day. Generally interview sessions with adults tended to last longer than those with target students or nominated peers.

Target students, parents, and teachers were asked each question three times in succession for each of the nominated peers. For example, the target student, Tom, was asked, 'Do you and Steve play together at lunch or recess? Do you and Mark play together at lunch and recess? Do you and Sam play together at lunch and recess?' Questions for peers were asked about the target student and at least one other friend who was identified by the peer at the beginning of the interview. A 3-point scale with responses of 'always', 'sometimes' and 'never' was used with all respondents and a response option of 'I don't know' was provided. This scale was simpler than the 5-point scales used in previous research, (Bukowski et al., 1994; Parker \& Asher, 1993; Wiener \& Schneider, 2002) but it was considered that more children would be able to offer responses. Some children responded to the initial question with either 'yes' or 'no'. If respondents initially answered the question with a response of 'yes', they were then asked if they engaged in the behaviour 'some of the time' or 'all of the time'. The 'don't know' response was coded as missing data for analytical purposes. It was recognised that the inclusion of a 'don't know' option would compromise the completeness of datasets, but the researchers considered this preferable to forcing a response from a respondent (such as a parent or teacher) who did not have knowledge of the information, thereby eliminating the need for guessing. Parents and teachers were asked questions from the standpoint of the target student. For example, parents were asked, 'Do your child and Mark play together?' and teachers were asked, 'Do Tom and Mark play together?'

Several further adaptations were used to attempt to assist all students to respond to the questionnaire. At the beginning of the interview, several sample questions were given to students and the different responses were explained and demonstrated at this time. In addition, photographs of nominated peers were shown to all target students during the interview to remind them of the peers they were discussing at that time. All children who could read (and adults) were given a written copy of the interview format to follow as the interviewer asked the questions.

\section{Data Analysis}

Prior to analysis, responses were converted into numerical scores ranging from 3 for Always, 2 for Sometimes, and 1 for Never. Initially, a score for each friendship dimension (i.e., Companionship, Validation and Caring, Help and Guidance, Intimate Exchange, Conflict, and Conflict Resolution) was calculated for both the short and full interview forms. A separate score for each dyad was calculated for each of the four respondents 
(target child, peer, teacher, parent), who had a complete dataset across all questions relevant to a given dimension. This was accomplished by calculating the mean of the responses to all relevant questions. If an interviewee failed to respond or responded 'I don't know' to a relevant question, their data were excluded. To aid in sorting of data into high and low scoring groups, a Mean Interview Score was then calculated by averaging the individual dimension score across all respondents who had a complete dataset. The Mean Interview Score was based on between 1 and 4 respondent scores, depending on the amount of incomplete data. Thus, in order to evaluate the consistency of respondent scores for each dyad, an average deviation was calculated. The consistency of responses across the participants will be addressed later in this article.

\section{Results}

\section{Overall Response Rates}

Sixty per cent of target students, $89 \%$ of peers, and $100 \%$ of teachers and parents successfully completed the short interview form. A further $40 \%$ of target students, $61 \%$ of peers, as well as $100 \%$ of teachers and parents completed the full interview form. Those children who failed to successfully complete even the short interview form had either poor communication skills (e.g., could only provide Yes/No answers), limited English or did not meet the criteria for reliability (see next section). Four parents stated that they had little knowledge of any of the nominated peers and really did not know much about their child's playmates at school. In these cases, a response of 'I don't know' was recorded for all questions except Question 2 that asked whether nominated peers and target students played together at each other's houses. These four parents were able to provide responses to this question.

\section{Questionnaire Reliability}

Three questions were repeated on the short interview form to assess the reliability of the responses of all target students and nominated peers. These questions were Question 1/4, 'Do you and play together at lunch or recess?', Questions 6/8 'Do you and like the same things?' and Questions 10/13 'Do you and fight?'. Adults were not given reliability questions as the researchers considered that adults were more than capable of understanding the questions. Interview questions were determined to be reliable if the child gave the same response for each pair of the three reliability questions for all three peers. Children's responses were excluded from the results if they did not achieve $100 \%$ reliability across the three questions for all dyads. The responses of one target student and one nominated peer were excluded from analysis as they did not meet the criteria. As an additional check of reliability, responses on similar questions were compared to determine consistency in answers on the full interview form. Although identical responses would not necessarily be anticipated, a pattern of similar responses would be expected. Question pairs, that were considered to be very similar to each other, included questions 34 and 46, and questions 51 and 52 . A mean of $58 \%$ of responses on similar pairs of questions were in total agreement while an additional mean of $37 \%$ of responses were only different by 1 point and a mean of $4 \%$ of responses differed by 2 points. Thus, a reasonably good degree of consistency was found across questions that were similar to each other.

\section{Multiple Respondents}

One index of the success of using multiple respondents was the presence of at least some complete datasets for a dyad, particularly when responses provided by target children 
were incomplete or absent. A complete dataset for a dimension was considered to be present when a respondent was able to rate all the questions on a 3-point scale and did not reply 'don't know' to any question. Data on the percentage of complete datasets for respondents in each dimension are presented in Table 2. As expected, a larger percentage of respondents completed the short interview form than the full interview form. It should be noted that low figures for adult participants do not represent failure to complete the interview questionnaire but, rather, the presence of Don't know responses. As mentioned previously, all teachers and parents completed all of the questions on the full interview form. Peers had the highest mean of complete datasets (82.4\%) across all dimensions on the short interview form while teachers had the highest rate of complete datasets (50\%) across all dimensions on the full interview form. Parents $(39.1 \%)$ had the lowest mean of complete datasets on the short interview form. Both parents $(12.8 \%)$ and target students (14.0\%) had low mean scores for complete datasets on the full interview form. While the low rate of completed datasets was anticipated for target students, it was not expected for parents. In both interview forms, parents consistently provided the most 'don't know' responses resulting in incomplete datasets. Despite this finding, the use of multiple respondents resulted in a mean of $97.3 \%$ of dyads with at least one respondent score across all dimensions for questions on the short interview form and a mean of $78 \%$ of dyads with at least one respondent score across all dimensions for questions in the full interview form.

The Mean Interview Score was the primary measure of every relationship dimension for each dyad. As previously noted, this was based on between one and four respondent scores, depending on the amount of incomplete data. There was some concern about this approach to calculating the Mean Interview Score. If there were large variations in the respondent scores, the validity of using the Mean Interview Score would be questionable, as the individual respondent scores available for a given dyad may have unduly influenced the mean. Conversely, if there were a reasonably high degree of consistency between individual respondent scores for dyads, confidence in the Mean Interview Score would be greater as it would be less influenced by the particular respondents who had contributed data.

\section{TABLE 2}

Percentage of Respondents with Complete Datasets

\begin{tabular}{|c|c|c|c|c|c|c|c|c|}
\hline Respondent & $\begin{array}{l}\text { Percentage } \\
\text { completing } \\
\text { form }\end{array}$ & $\begin{array}{l}\text { Companion- } \\
\text { ship }\end{array}$ & $\begin{array}{l}\text { Validation } \\
\text { and } \\
\text { caring }\end{array}$ & $\begin{array}{c}\text { Help } \\
\text { and } \\
\text { guidance }\end{array}$ & $\begin{array}{l}\text { Intimate } \\
\text { exchange }\end{array}$ & Conflict & $\begin{array}{l}\text { Conflict } \\
\text { resolution }\end{array}$ & $\begin{array}{c}\text { Mean } \\
\text { across } \\
\text { dimensions }\end{array}$ \\
\hline \multicolumn{9}{|l|}{$\begin{array}{l}\text { Short interview } \\
\text { form }\end{array}$} \\
\hline Target student & 59.5 & 59.5 & 45.9 & 54.1 & 56.8 & 58.1 & & 54.9 \\
\hline Peer & 89.2 & 89.2 & 68.9 & 78.4 & 87.8 & 87.8 & & 82.4 \\
\hline Teacher & 100.0 & 66.2 & 71.6 & 86.5 & 75.1 & 89.2 & & 77.7 \\
\hline Parent & 100.0 & 48.6 & 29.7 & 32.4 & 29.7 & 55.4 & & 39.2 \\
\hline \multicolumn{9}{|l|}{$\begin{array}{l}\text { Full interview } \\
\text { form }\end{array}$} \\
\hline Target student & 20.3 & 15.0 & 9.5 & 10.8 & 18.9 & 17.6 & 12.2 & 14.0 \\
\hline Peer & 60.8 & 52.7 & 33.8 & 37.8 & 58.1 & 55.4 & 40.5 & 46.4 \\
\hline Teacher & 100.00 & 59.5 & 23.0 & 55.4 & 40.5 & 74.3 & 47.3 & 50.0 \\
\hline Parent & 100.00 & 16.2 & 2.7 & 8.1 & 12.2 & 27.0 & 10.8 & 12.8 \\
\hline
\end{tabular}


Thus, in order to evaluate the consistency of respondent scores for each dyad, an average deviation was calculated for all dyads with multiple respondent scores. The average deviation was calculated by taking the mean of the absolute difference between each respondent score and the average, for all respondents for a given dyad. For dyads with three respondent scores, the maximum average deviation possible was .88 and for dyads with two or four respondent scores, the maximum average deviation possible was 1.00. A mean average deviation score of $0.30(S D=0.06$, range $0.23-0.39)$ was calculated across all dimensions in the short interview form and a mean average deviation of 0.25 $(S D=0.05$, range 0.18-0.32) was calculated across all dyads and dimensions in the full interview form. These values indicate that respondent scores on the short interview form for all dimensions had a fairly high degree of consistency and, thus, there was reasonable justification in using Mean Interview Scores. In addition, mean average deviation scores for all dimensions in the full interview form except Conflict Resolution (0.32) and all dimensions in the short interview form except Help and Guidance (0.39) were below 0.30 indicating a fairly high degree of consistency across multiple respondent scores. It should be reiterated that some difference in perception among multiple respondents was expected.

\section{Short and Full Interview Forms}

Another important methodological adaptation was the development of the short interview form to accommodate those children who were not able to complete the full interview form of the questionnaire. Thus, it was important to determine how closely the results of the two forms corresponded. The Mean Interview Score across each dimension for the short and full interview forms are presented in Figure 1. Both short and full interview forms evidenced very similar patterns across the dimensions of relationships. It should be noted that Conflict Resolution was not included in this analysis as no question for this dimension was present on the short interview form. In order to further investigate the relationship between short and full interview forms, Pearson product moment correlations were calculated for students who had complete datasets for both interview forms. Correlations for individual dimensions were 0.76 for

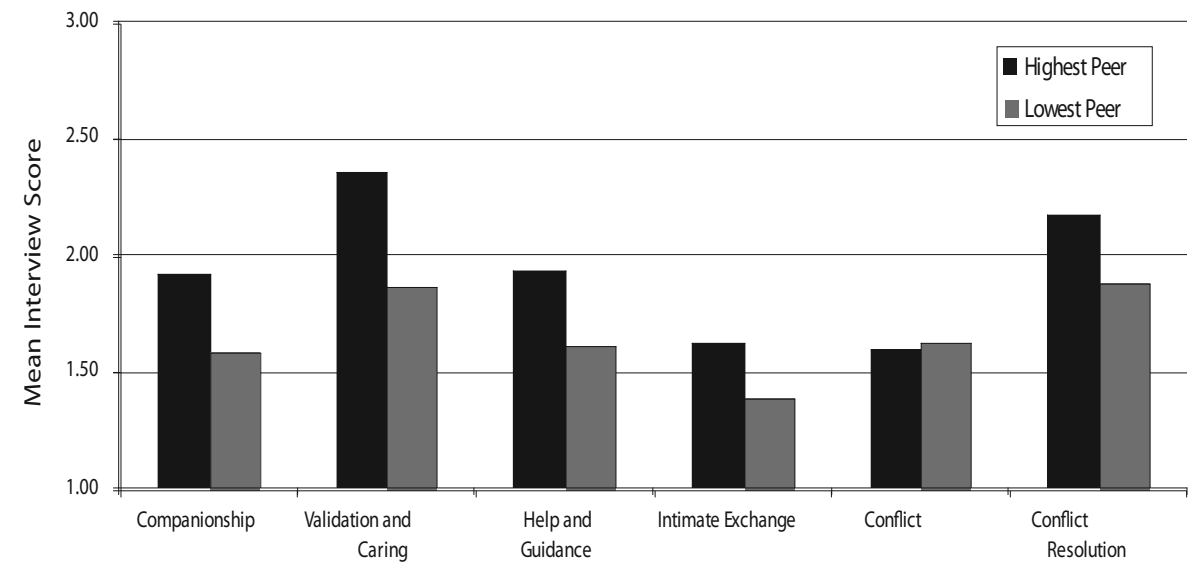

Dimension 
Conflict, 0.69 for Validation and Caring, 0.68 for Help and Guidance, 0.65 for Companionship and 0.46 for Intimate Exchange. Correlations were found to be high for all dimensions except Intimate Exchange, which had only a moderate correlation. Thus, results from the short interview form were generally similar to those obtained on the full interview form for each dimension. It should be noted that as each target student contributed multiple times to the dataset, inferential procedures were not considered to be appropriate.

\section{Discussion}

The primary objective of the current study was to determine whether a questionnaire protocol previously used with typically developing children could be adapted to describe the school-based close relationships of children with developmental disabilities. The adaptations included the use of multiple respondents and the development of a shortform questionnaire. Adaptations were also made to procedures in order to accommodate the chronological and developmental levels of the target students and peers. These included simplification of the response scale to a 3-point scale and the use of photographs of peers during interviews with target students.

One of the general indices of the success of the adaptations was the number of students who completed the questionnaire. Sixty percent of target students and $89 \%$ of peers successfully completed the short interview form, while $40 \%$ of target students and $61 \%$ of peers also completed the full interview form. Further evidence was provided in the level of reliability of child responses. Data from only one target student and one peer were excluded from analysis as they did not meet the reliability criteria. Supplementary analysis also suggested high correspondence between responses to similar questions. Thus, in general terms, the adaptations can be considered successful in obtaining reliable data from a relatively high percentage of child respondents.

A particular and important adaptation was the use of the short interview form to cater for younger students and those students with communication difficulties who were not able to complete the full interview form. One indication of the success of this adaptation was the correspondence of results between the short and full interview forms for most dimensions. Correlations for all dimensions were above 0.65 with the exception of Intimate Exchange (0.46). Although the longer interview form may provide more comprehensive information, the shorter format provides generally consistent information on the dimensions of children's relationships. In addition, the use of the short interview form was found to be extremely valuable in obtaining data from a substantial number of target students and peers for whom the full interview form was not appropriate.

Despite the inclusion of a shorter version of the questionnaire, it was anticipated that some of the children involved would not be able to attempt even the short interview form. Thus, in order to gain the most complete information on each dyad, interviews were conducted with multiple respondents (i.e., target student, peer, parent, and teacher). Previous research with both typically developing children and children with disabilities (Bauminger \& Kasari, 2000; Bukowski et al., 1994; Heiman, 2000; Parker \& Asher, 1993; Wiener \& Schneider, 2002) has included interviews with the target student and sometimes the peer. Some researchers (Buysse, 1993; Guralnick et al., 1995) who have studied friendships of very young children with disabilities have interviewed the mothers of these students, but not the children. The propriety of this approach is uncertain as it may not have reflected the views of the children involved in the 
relationship. In contrast with the approach to interviews adopted in previous research, the present study included interviews with both children and adults (i.e., specifically teachers and parents).

Although some disparity in the answers of multiple respondents was anticipated, mean average deviation scores indicated a relatively high degree of consistency between respondents in most instances. Further, valuable data were obtained on relationships that would not otherwise have been revealed because children's age or communicative limitations prohibited them from successfully completing the interview. A mean of $97.3 \%$ of dyads had at least one respondent score across all dimensions for the short interview form and a mean of $78 \%$ of dyads had at least one respondent score across all dimensions for the full interview form. In summary, the use of interviews across multiple respondents appeared to have been an effective method of providing comprehensive and multifaceted information about the relationships of dyads.

In the present study, respondents were offered the option to answer 'don't know' to questions about which they were uncertain. In contrast, previous researchers have typically forced a response from participants (e.g., Bukowski et al., 1994; Parker \& Asher, 1993; Wiener \& Schneider, 2002). Although, as anticipated, the inclusion of this option resulted in a fairly high number of incomplete datasets from some respondent groups, incomplete data was regarded as preferable to misleading data that might result from respondents' guessing, particularly noting that parents and teachers may not be in a position to have knowledge of some aspects of relationships. The prevalence of 'don't know' answers for certain respondents, particularly parents, highlights a possible flaw in some previous research that has relied on the forced responses of one participant, when they might not necessarily have had the information to answer.

A number of limitations of the present study should be acknowledged. One important limitation, shared by most of the extant research, was that the data reported were exclusively derived from interviews. Respondents to interviews, and children in particular, may not necessarily report behaviours accurately (Furman, 1996; Wiener \& Schneider, 2002). While a technically challenging task, it would be useful in future research to attempt to verify interview data with direct observation. Furthermore, in the present study, data from the full interview form were often incomplete. Many children were not able to answer all relevant questions for individual dimensions. In addition, some parents had little knowledge of their child's relationships at school. Nevertheless, the use of the short interview form and multiple respondents did compensate to some extent for the lack of complete datasets in the full interview form.

In conclusion, the current study investigated the success of adaptations to methodology previously used with typically developing children in order to examine relationships between children with developmental disabilities and peers. These adaptations included the use of multiple respondents in interview sessions, changes to the interview scale and the creation of an alternative short interview form. In general these adaptations were successful in eliciting information on aspects of children's relationships that might not have been obtained using the traditional interview instrument and were able to compensate for the communication difficulties and age ranges of the target students. In addition, the perspectives of peers, parents and teachers were also obtained that contributed to the overall information on children's relationships. Thus, the approach described in this paper may represent a useful addition to existing methodologies for examining relationships in children with developmental disabilities in inclusive settings. This may open the way for further detailed research on relationships of these children. 


\section{References}

Asher, S.R., Parker, J.G., \& Walker, D.L. (1996). Distinguishing friendship from acceptance: Implications for intervention and assessment. In W.M. Bukowski, A.F. Newcomb, \& W.W. Hartup (Eds.), The company they keep: Friendship in childhood and adolescence (pp. 366-405). New York: Cambridge University Press.

Bauminger, N., \& Kasari, C. (2000). Loneliness and friendship in high-functioning children with autism. Child Development, 71, 447-456.

Berndt, T.J., \& Perry, T.J. (1986). Children's perceptions of friendships as supportive relationships. Developmental Psychology, 22, 640-648.

Bhasin, T.K., Brocksen, S., Avchen, R.N., \& Braun, K.V.N. (2006). Prevalence of four developmental disabilities among children aged 8 years. Metropolitan Atlanta Developmental Disabilities Surveillance Program, 1996 and 2000. Retrieved 10 July, 2008, from http://www.cdc.gov/ $\mathrm{mmwR} /$ preview/mmwrhtml/ss5501al.htm\#tab1

Bukowski, W.M., Hoza, B., \& Boivin, M. (1994). Measuring friendship quality during pre and early adolescence: The development and psychometric properties of the friendship qualities scale. Journal of Social and Personal Relationships, 11, 471-484.

Bukowski, W.M., Hoza, B., \& Newcomb, A.F. (1987). Friendship, popularity, and the "self" during adolescence.Unpublished manuscript, University of Maine, Department of Psychology.

Buysse, V. (1993). Friendships of preschoolers with disabilities in community-based child care settings. Journal of Early Intervention, 17, 380-395.

Buysse, V., \& Bailey, D.B., Jr. (1993). Behavioral and developmental outcomes in young children with disabilities in integrated and segregated settings: A review of comparative studies. The Journal of Special Education, 26, 434-461.

Buysse, V., Goldman, B.D., \& Skinner, M.L. (2002). Setting effects on friendship formation among young children with and without disabilities. Exceptional Children, 68, 503-517.

Buysse, V., Nabors, L., Skinner, D., \& Keyes, L. (1997). Playmate preferences and perceptions of individual differences among typically developing preschoolers. Early Child Development and Care, $131,1-18$.

Centre for Developmental Disability Studies. (2001). What is developmental disability. Retrieved August 12, 2003, from http://www.cdds.med.usyd.edu.au/html/what_is_dd.html

Chamberlain, B., Kasari, C., \& Rotherham-Fuller, E. (2007). Involvement or isolation? The social networks of children with autism in regular classrooms. Journal of Autism and Developmental Disorders, 37, 230-242.

Cuckle, P., \& Wilson, J. (2002). Social relationships and friendships among young people with Down's syndrome in secondary schools. British Journal of Special Education, 29, 66-71.

Developmental Disabilities Assistance and Bill of Rights Act. (2000). Retrieved 11 August, 2003 from www.act.dhhs.gov/programs/add/DD-ACT2.htm.

Evans, I.M., Salisbury, C.L., Palombaro, M.M., Berryman, J., \& Hollowood, T.M. (1992). Peer interactions and social acceptance of elementary-age children with severe disabilities in an inclusive school. Journal of the Association for Persons with Severe Handicaps, 17, 205-212.

Falvey, M., \& Rosenberg, R.L. (1995). Developing and fostering friendships. In M.A. Falvey (Ed.), Inclusive and heterogeneous schooling: Assessment, curriculum, and instruction (pp. 267-283). Baltimore: Paul H. Brookes.

Field, T. (1984). Play behaviors of handicapped children who have friends. In T. Field, J.L. Roopnarine, \& M. Segal (Eds.), Friendships in normal and handicapped children (pp. 153-162). Norwood, NJ: Ablex Publishing Corporation.

Fisher, M. (2001). Andre's story: Frames of friendship. In M. Grenot-Scheyer, M. Fisher, \& D. Staub (Eds.), Lessons learned in inclusive education (pp. 91-111). Baltimore: Paul Brookes.

Freeman, S.F.N. (2000). Academic and social attainments of children with mental retardation in general education and special education settings. Remedial and Special Education, 21, 3-20.

Freeman, S.F. N., \& Kasari, C. (1998). Friendships in children with developmental disabilities. Early Education and Development, 9, 341-355. 
Fujiki, M., Brinton, B., Hart, C.H., \& Fitzgerald, A.H. (1999). Peer acceptance and friendship in children with specific language impairment. Topics in Language Disorders, 19(2), 34-48.

Furman, W. (1996). The measurement of friendship perceptions: Conceptual and methodological issues. In W.M. Bukowski, A.F. Newcomb, \& W.W. Hartup (Eds.), The company they keep: Friendship in childhood and adolescence (pp. 41-65). New York: Cambridge University Press.

Gottman, J.M. (1983). How children become friends. Monographs for the Society for Research in Child Development, 48(3, Serial No. 201).

Grenot-Scheyer, M., Fisher, M., \& Staub, D. (2001). A framework for understanding inclusive education. In M. Grenot-Scheyer, M. Fisher, \& D. Staub (Eds.), Lessons learned in inclusive education (pp. 118). Baltimore: Paul H. Brookes Publishing Company.

Guralnick, M.J. (1997). Peer social networks of young boys with developmental delays. American Journal on Mental Retardation, 101, 595-612.

Guralnick, M J. (1999). The nature and meaning of social integration for young children with mild developmental delays in inclusive settings. Journal of Early Intervention, 22, 70-86.

Guralnick, M.J., Connor, R.T., \& Hammond, M. (1995). Parent perspectives of peer relationships and friendships in integrated and specialized programs. American Journal on Mental Retardation, 99, 457-476.

Guralnick, M.J., \& Groom, J.M. (1988). Friendships of preschool children in mainstreamed playgroups. Developmental Psychology, 24, 595-604.

Hall, L.J. (1994). A descriptive assessment of social relationships in integrated classrooms. Journal of the Association for Persons with Severe Handicaps, 19, 302-313.

Hall, L.J., \& Strickett, T. (2002). Peer relationships of preadolescent students with disabilities who attend a separate school. Education and Training in Mental Retardation and Developmental Disabilities, 37, 399-409.

Hamre-Nietupski, S. (1993). How much time should be spent on skill instruction and friendship development? Preferences of parents of students with moderate and severe/ profound disabilities? Education and Training in Mental Retardation and Developmental Disabilities, 28, 220-231.

Hamre-Nietupski, S., Hendrickson, J., Nietupski, J., Sasson, G., \& Shokoohi-Yekta, M. (1993). Perceptions of teachers of students with moderate, severe, or profound disabilities on facilitating friendships with nondisabled peers. Education and Training in Mental Retardation and Developmental Disabilities, 28, 111-127.

Heiman, T. (2000). Friendship quality among children in three educational settings. Journal of Intellectual \& Developmental Disability, 25, 1-12.

Howes, C. (1983). Patterns of friendship. Child Development, 54, 1041-1053.

Hurley-Geffner, C. M. (1995). Friendships between children with and without developmental disabilities. In R.L. Koegel \& L.K. Koegel (Eds.), Teaching children with autism (pp. 105-125). Baltimore: Paul H. Brookes.

Kennedy, C.H., \& Itknonen, T. (1994). Some effects of regular class participation on the social contacts and social networks of high school students with severe disabilities. Journal of the Association for Persons with Severe Handicaps, 19, 1-10.

Ladd, G.W., Kochenderfer, B.J., \& Coleman, C.C. (1996). Friendship quality as a predictor of young children's early school adjustment. Child Development, 67, 1103-1118.

Lee, S.H., Yoo, S.Y., \& Bak, S.H. (2003). Characteristics of friendships between children with and without mild disabilities. Education and Training in Mental Retardation and Developmental Disabilities, 38, 157-166.

Mannarino, A.M. (1980). The development of children's friendships. In H.C. Foot, A.P. Chapman, \& J.R. Smith (Eds.), Friendship and social relations in children (pp. 45-63). New York: John Wiley and Sons.

Meyer, L.H., Minondo, S., Fisher, M., Larson, M.J., Dunmore, S., Black, J.W., et al. (1998). Frames of friendship: Social relationships among adolescents with diverse abilities. In L.H. Meyer, H.-S. Park, M. Grenot-Scheyer, I.S. Schwartz, \& B. Harry (Eds.), Making friends: The influences of culture and development (pp. 189-221). Baltimore: Paul H. Brookes.

Moore, C. (1998). Educating students with disabilities in general education classrooms: A summary of the research. Retrieved July 8, 2003, from http://interact.uoregon.edu/wrrc/AKInclusion.html 
Nabors, L. (1997). Playmate preferences of children who are typically developing for their classmates with special needs. Mental Retardation, 35, 107-113.

National Association of Developmental Disabilities Councils. (2003). What is a developmental disability? Retrieved August 11, 2003, from http://www.naddc.org/DD\%20Basics.htm

Newcomb, A.F., \& Bagwell, C.L. (1996). The developmental significance of children's friendship relations. In W. M. Bukowski, A.F. Newcomb, \& W.W. Hartup (Eds.), The company they keep: Friendship in childhood and adolescence (pp. 289-321). New York: Cambridge University Press.

Northern Territory Government. (2005). Central Region: Social-economic snapshot. Retrieved 23 July, 2007 from http://www.nt.gov.au/business/services.cfm?cat4id=14

Overton, S., \& Rausch, J.L. (2002). Peer relationships as support for children with disabilities: An analysis of mothers' goals and indicators for friendship. Focus on Autism and Other Developmental Disabilities, 17, 11-20.

Parker, J.G., \& Asher, S.R. (1993). Friendship and friendship quality in middle childhood: Links with peer group acceptance and feelings of loneliness and social dissatisfaction. Developmental Psychology, 29, 611-621.

Rice, C. (2007). Prevalence of autism spectrum disorders - Autism and developmental disabilities monitoring network, 14 Sites, United States, 2002. Retrieved 10 July, 2008, from http://www.cdc.gov/MMWR/preview/mmwrhtml/ss5601a2.htm

Richardson, P., \& Schwartz, I.S. (1998). Making friends in preschool: Friendship patterns of young children with disabilities. In L.H. Meyer, H.-S. Park, M. Grenot-Scheyer, I.S. Schwartz, \& B. Harry (Eds.), Making friends: The influences of culture and development (pp. 65-80). Baltimore: Paul H. Brookes.

Salisbury, C.L., \& Palombaro, M.M. (1998). Friends and acquaintances: Evolving relationships in an inclusive elementary school. In L.H. Meyer, H.-S. Park, M. Grenot-Scheyer, I.S. Schwartz, \& B. Harry (Eds.), Making friends: The influences of culture and development (pp. 81-104). Baltimore, MD: Paul H. Brookes.

Schneider, B.H. (1999). A mutimethod exploration of the friendships of children considered socially withdrawn by their school peers. Journal of Abnormal Child Psychology, 28(2), 115-123.

Schneider, B.H., Fonzi, A., Tani, F., \& Tomada, G. (1997). A cross-cultural exploration of the stability of children's friendships and the predictors of their continuation. Social Development, 6, 322-339.

Siperstein, G.N., Leffert, J.S., \& Wenz-Gross, M. (1997). The quality of friendships between children with and without learning problems. American Journal on Mental Retardation, 102, 111-125.

Sparrow, S.S., Balla, D.A., \& Cicchetti, D.V. (1985). Vineland adaptive behavior scales: Classroom edition. Circle Pines, MN: American Guidance Service, Inc.

Staub, D. (1998). Delicate threads: Friendships between children with and without disabilities. Bethesda, MD: Woodbine House.

Strain, P.S. (1984). Social behavior patterns of nonhandicapped and developmentally disabled friend pairs in mainstream preschools. Analysis and Intervention in Developmental Disabilities, 4, 15-28.

Villa, R.A., \& Thousand, J.S. (1995). The rationales for creating inclusive schools. In R.A. Villa, \& J.S. Thousand (Eds.), Creating an inclusive school (pp. 28-44). Alexandria, VA: Association for Supervision and Curriculum Development.

Webster, A.A., \& Carter, M. (2007). Social relationships and friendships of children with developmental disabilities: Implications for inclusive settings. A systematic review. Journal of Intellectual \& Developmental Disability, 32, 200-213.

Wiener, J., \& Schneider, B.H. (2002). A multisource exploration of the friendship patterns of children with and without learning disabilities. Journal of Abnormal Child Psychology, 30, 127-141.

Wiener, J., \& Tardif, C.Y. (2004). Social and emotional functioning of children with learning disabilities: Does special education make a difference? Learning Disabilities Research \& Practice, 19, 20-32.

Zetlin, A.G., \& Murtaugh, M. (1988). Friendship patterns of mildly learning handicapped and nonhandicapped high school students. American Journal on Mental Retardation, 92, 447-454. 